\title{
FOXM1 is a novel predictor of recurrence in patients with oral squamous cell carcinoma associated with an increase in epithelial-mesenchymal transition
}

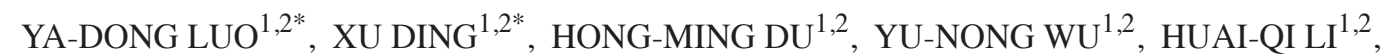 \\ HE-MING WU ${ }^{1,2}$ and XIAO-MIN ZHANG ${ }^{1,3}$ \\ ${ }^{1}$ Jiangsu Key Laboratory of Oral Diseases; Departments of ${ }^{2}$ Oral and Maxillofacial Surgery and ${ }^{3}$ Paediatric Dentistry, \\ Affiliated Hospital of Stomatology, Nanjing Medical University, Nanjing, Jiangsu 210029, P.R. China
}

Received June 14, 2018; Accepted January 22, 2019

DOI: $10.3892 / \mathrm{mmr} .2019 .10094$

\begin{abstract}
Although forkhead box protein M1 (FOXM1) is markedly upregulated in human premalignant and oral squamous cell carcinoma (OSCC) tissues and cultured cells, the association of FOXM1 expression with OSCC prognosis is not well understood. The present study investigated the possible association of FOXM1 expression in patients with OSCC with their clinicopathological characteristics and clinical outcomes. The expression of FOXM1 protein in OSCC tissues from 119 patients was evaluated by immunohistochemistry, and the results demonstrated that FOXM1 overexpression in patients with OSCC was associated with tumour recurrence and poor prognosis. To study the in vitro effects of FOXM1, its expression was decreased by small interfering RNA (siRNA) in OSCC cell lines, and FOXM1 knockdown decreased the proliferative, migratory and invasive capacities of cells. FOXM1 inhibition by siRNA gave rise to reduced expression of vimentin and increased expression of E-cadherin. The present study reported FOXM1 as a novel predictor of tumour recurrence in patients with OSCC and its potential involvement in epithelial-mesenchymal transition in OSCC cells.
\end{abstract}

\section{Introduction}

Oral squamous cell carcinoma (OSCC) is among the 10 most common cancer types worldwide and represents $\sim 90 \%$ of

Correspondence to: Dr Xiao-Min Zhang or Professor He-Ming Wu, Jiangsu Key Laboratory of Oral Diseases, Nanjing Medical University, 136 Hanzhong Road, Nanjing, Jiangsu 210029, P.R. China

E-mail: zhangxiaomin2018@aliyun.com

E-mail:whmz2002@aliyun.com

\section{${ }^{*}$ Contributed equally}

Key words: oral squamous cell carcinoma, forkhead box protein M1, epithelial-mesenchymal transition, recurrence, prognosis malignant tumours in the oral cavity (1). Although considerable advances in oral cancer detection, prevention and treatment have been made over the past 20 years, the 5-year survival rate of OSCC remains $\sim 50 \%(2,3)$. OSCC treatment failures are primarily caused by local/regional tumour recurrence, and postoperative tumour recurrence leads to poor prognosis and poor quality of life. An improved understanding of the mechanism of local recurrence may lead to the development of new diagnostic and prognostic criteria and novel therapeutic targets.

According to the World Health Organization (WHO) lineage of origin classification, the transcription factor forkhead box (FOX) protein M1 (FOXM1) belongs to the FOX protein family $(4,5)$. FOXM1 serves important roles in cell cycle progression and mitosis during embryonic development and in adult tissue homeostasis, and recent studies have indicated that FOXM1 serves a pivotal role in the development and evolution of a number of human cancer types (6-9). FOXM1 is not only a key cell cycle regulator, but is also critical to tumorigenesis, tumour aggressiveness and metastasis (4,10-12). The upregulation of FOXM1 has been identified in cancer, including lung cancer, basal cell carcinoma, pancreatic cancer, and head and neck squamous cell carcinoma (HNSCC) $(13,14)$. Notably, in breast cancer, gastric cancer and laryngeal squamous cell carcinoma, high expression of FOXM1 is associated with poor prognosis (15-17). Although FOXM1 is markedly upregulated in HNSCC tissues and cultured cells, the association of FOXM1 expression with OSCC prognosis has yet to be fully elucidated.

The present study examined the expression of FOXM1 in 119 primary OSCCs by immunohistochemistry (IHC) and analysed its potential associations with clinical and pathological characteristics.

\section{Patients and methods}

Patients and specimens. All 119 patients with stage I-IV OSCC included in the present study were initially diagnosed and treated at the Department of Oral and Maxillofacial Surgery, Affiliated Stomatological Hospital of Nanjing Medical University (Nanjing, China), between January 2005 and December 2008. None of the patients received correlative treatment prior to the first pathological examination. All patients 
were diagnosed as SACC according to clinical and pathological examination and underwent radical resection without any previous radiation or chemotherapy. The tumours were diagnosed based on the International Union Against Cancer tumour size, nodal metastasis and distant metastasis (TNM) classification (18). The histological results were confirmed based on the WHO definitions of the tumour types. Following diagnosis, all the patients underwent radical surgery. To evaluate postoperative conditions, the patients were re-examined every 3 months for the first 2 years and every 6 months for the next 3 years. The present study was performed with permission from the ethical committee of the Affiliated Stomatological Hospital of Nanjing Medical University. Informed consent was obtained from each patient. The follow-up period was 3-81 months (mean, 37.3 months). Local recurrence following surgery was confirmed by pathological biopsy.

Assessment of FOXM1 expression in OSCC by IHC. The tissues were fixed in formalin overnight at $4^{\circ} \mathrm{C}$, embedded in paraffin and cut into $4-\mu \mathrm{m}$ sections. The sections were deparaffinized through a graded alcohol series and incubated in $3 \% \mathrm{H}_{2} \mathrm{O}_{2}$ at room temperature for $20 \mathrm{~min}$ to block endogenous peroxidases. The sections were microwaved in citrate buffer $(\mathrm{pH} 6.0)$ for $20 \mathrm{~min}$ for antigen retrieval and subsequently incubated with $1 \%$ bovine serum albumin (BSA; Sigma-Aldrich; Merck KGaA, Darmstadt, Germany) in phosphate-buffered saline (PBS) for $1 \mathrm{~h}$ at room temperature to block nonspecific binding. The sections were incubated overnight at $4^{\circ} \mathrm{C}$ with anti-FOXM1 (rabbit polyclonal antibody; 1:500; ab55006; Abcam, Cambridge, UK) and rinsed a number of times with PBS, and sections incubated with $1 \%$ BSA were used as negative controls. Following sequential incubation with the corresponding anti-rabbit secondary antibodies (1:200; ab207298; Abcam) for $30 \mathrm{~min}$ at $37^{\circ} \mathrm{C}$ and avidin-biotin-peroxidase reagent for $30 \mathrm{~min}$, the signals were visualized using a diaminobenzidine staining kit (DAKOCytomation; Agilent Technologies, Inc., Santa Clara, CA, USA) for $3 \mathrm{~min}$ at room temperature. The OSCC tissue sections were subsequently counterstained with $0.5 \%$ haematoxylin for $5 \mathrm{~min}$ at room temperature.

The immunoreactivity of FOXM1 was assessed based on the cytoplasmic and nuclear staining. The staining intensity was categorized as follows: 0 , negative; 1 , weak; 2 , moderate; and 3 , intense. A score based on the proportion of cells with positive expression was obtained according to the following criteria: 0,$0 ; 1,1-10 ; 2,11-50 ;$ and $3,>50 \%$. The staining intensity was multiplied by the proportion scores to obtain a comprehensive score. Comprehensive scores exceeding 5 represented high FOXM1 expression, whereas other scores indicated low FOXM1 expression. The scores of the different cases were analysed independently by two pathologists who had no knowledge of the clinical data. In almost all cases, the differences between the scores of each pathologist were $<10 \%$.

Cell culture. The human OSCC cell lines HN4, HN6, HN12, Tca-811 and SCC-22B (generously provided by Shanghai Ninth People's Hospital, Shanghai, China), which had been used in previous study (19-23). HN4, HN6, HN12, Tca-811 and SCC-22B and Cal-27 (purchased from American Type Culture Collection, Manassas, VA, USA) were incubated in Dulbecco's modified Eagle's medium (DMEM, Gibco; Thermo Fisher Scientific, Inc., Waltham, MA, USA) containing 10\% foetal bovine serum (FBS; Gibco; Thermo Fisher Scientific, Inc.) at $37^{\circ} \mathrm{C}$ in a $5 \% \mathrm{CO}_{2}$ incubator.

Small interfering RNA and transfection. The FOXM1 small-interfering RNA (siRNA) sequence was designed according to a previous publication (24): 5-GGACCACUU UUCCCUACUUUDTDT-3 (sense) and 5-AAAGUAGGG AAAGUGGUCCDTDT-3 (antisense). The sequences of the scrambled siRNA were 5-UUCUCCGAACGUGUCACG UDTDT-3 (sense) and 5-ACGUGACACGUUCGGAGAADT DT-3 (antisense). RNA oligonucleotides were purchased from Shanghai GenePharma Co., Ltd. (Shanghai, China). $3 \mu 1$ Lipofectamine ${ }^{\circledR} 2000$ (Invitrogen; Thermo Fisher Scientific, Inc.) was used for the transfection of the 100 pmol siRNAs per six-well plate in accordance with the manufacturer's protocol.

Cell proliferation assay. Briefly, the cells were transiently transfected with scrambled siRNA and FOXM1 siRNA and, $24 \mathrm{~h}$ later, the cells were plated in 96-well plates $\left(5 \times 10^{3}\right.$ cells/well) and incubated for $72 \mathrm{~h}$ at $37^{\circ} \mathrm{C}$. The cells were fixed with $50 \mu \mathrm{l}$ of $50 \%$ pre-cooled trichloroacetic acid per well at $4^{\circ} \mathrm{C}$ for $1 \mathrm{~h}$ and washed five times with PBS. The cells were stained with $100 \mu \mathrm{l}$ of $0.4 \%$ sulforhodamine B (SRB) solution (Sigma-Aldrich; Merck KGaA) per well for 15 min at room temperature and washed five times with $1 \%$ acetic acid. Protein-bound SRB was mixed with $150 \mu \mathrm{l}$ of $10 \mathrm{mmol} / \mathrm{l}$ Tris-based solution and agitated for $5 \mathrm{~min}$. The optical densities at $560 \mathrm{~nm}$ of the samples in six parallel wells were measured using a multi-well spectrophotometer (VERSAmax, Molecular Devices, LLC, Sunnyvale, CA, USA).

Wound healing assay. OSCC cells were plated in a six-well plate and FOXM1 was knocked down. After $24 \mathrm{~h}$, the cells were seeded in 24-well plates in $10 \%$ serum-containing media, incubated until they reached $100 \%$ confluence, serum-starved for 1 day and wounded with a 200- $\mu 1$ plastic tip. The cells were washed three times with sterile PBS to remove the floating cells and incubated for $24 \mathrm{~h}$. The same wound areas were observed and imaged under a light microscope (magnification, x200; Leica Microsystems GmbH, Wetzlar, Germany) at 0 and 24 h.

Cell migration assay. OSCC cells were plated in a six-well plate and FOXM1 was knocked down. After 24 h, cell suspensions were prepared and counted. Subsequently, $5 \times 10^{4}$ cells in $100 \mu \mathrm{l}$ of serum-free media were seeded in each upper chamber of the Transwell inserts $(10-\mu \mathrm{m}$ thickness and $8-\mu \mathrm{m}$ pore size), and $600 \mu \mathrm{l}$ of DMEM with $10 \%$ FBS was added to each lower chamber. The cells were cultured in 24-well Transwell chambers for 12 and $72 \mathrm{~h}$ at $37^{\circ} \mathrm{C}$ in an atmosphere of $5 \% \mathrm{CO}_{2}$, and the cells from the upper chamber were gently removed. The cells that had invaded the lower chamber were fixed with $5 \%$ glutaraldehyde $30 \mathrm{~min}$ at room temperature and stained with $0.1 \%$ crystal violet for $3 \mathrm{~min}$ at room temperature. The stained cells were observed under an inverted microscope (magnification, x200) and repeatedly counted three times.

Western blot analysis. OSCC cells were plated in six-well plates and FOXM1 was knocked down. The cells were 

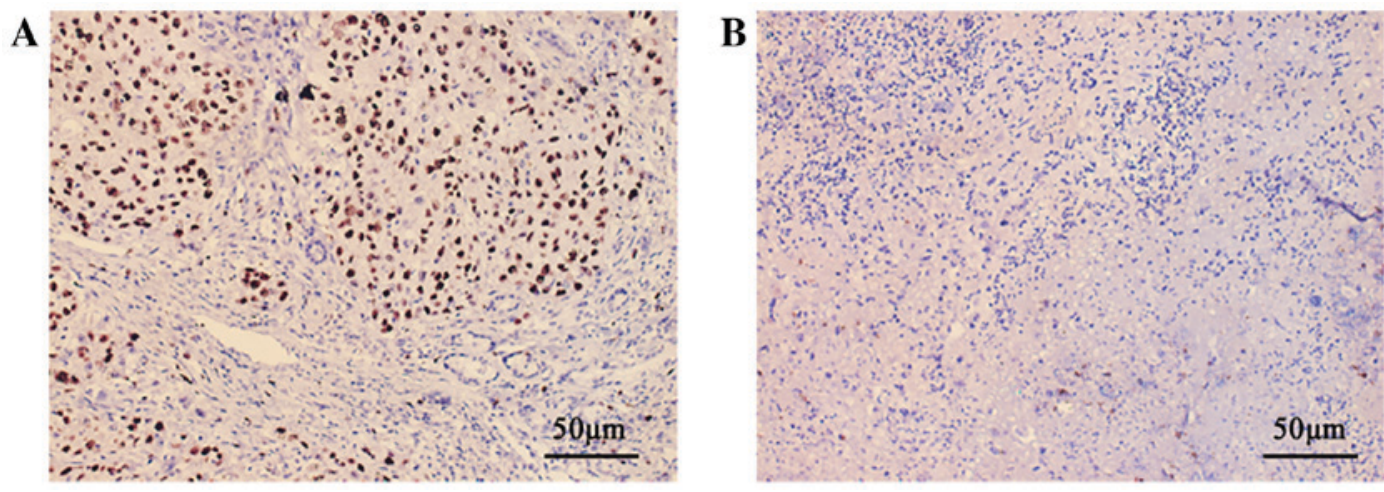

Figure 1. FOXM1 expression in oral squamous cell carcinoma by immunohistochemistry. Detection of (A) positive and (B) negative FOXM1. FOXM1, forkhead box protein M1.

collected, suspended in lysis buffer $(50 \mathrm{mmol} / 1 \mathrm{Tris}-\mathrm{HCl}$, $\mathrm{pH} 6.8,100 \mathrm{mmol} / 1 \mathrm{DTT}, 2 \% \mathrm{SDS}, 0.1 \%$ bromophenol blue and $10 \%$ glycerol) on ice for 5-10 min and centrifuged at $4^{\circ} \mathrm{C}$ $(12,000 \times \mathrm{g}, 15 \mathrm{~min})$. The protein concentrations were determined using a bicinchoninic acid protein assay. The $50 \mu \mathrm{g}$ protein samples per lane were separated by $10 \%$ SDS-PAGE and transferred onto nitrocellulose filter membranes. The membranes were blocked in freshly prepared PBS containing $5 \%$ non-fat dried milk for $2 \mathrm{~h}$ at room temperature. The blots were subsequently probed with primary antibodies specific for FOXM1, E-cadherin, vimentin and $\beta$-actin $(1: 1,000$; ab55006, ab1416, ab45939 and ab8226 respectively; Abcam,) overnight at $4^{\circ} \mathrm{C}$. The membranes were washed three times with PBS- $0.05 \%$ Tween 20 and incubated with the corresponding secondary antibodies (1:1,000; ab6940; Abcam) for $1 \mathrm{~h}$ at room temperature. The blots were subsequently incubated in the dark for enhanced chemiluminescence and visualized through exposure to ECL reagents (GE Healthcare, Chicago, IL, USA).

Statistical analysis. Data were tabulated, and statistical analyses were performed using SPSS 22.0 statistical software (IBM Corp., Armonk, NY, USA). The statistical significance between all comparisons of clinicopathological features was examined using $\chi^{2}$ analysis. The time from the first diagnosis date to the date of mortality or the last day of the follow-up assessment was plotted as the overall survival time. The period from therapy to local recurrence or metastasis was considered the period of disease-free survival. The survival rate curves were analysed using the Kaplan-Meier method, and the log-rank test was used to evaluate the significance of the differences. The significance of variables for survival rates was analysed using the Cox proportional hazards model in univariate and multivariate analyses. The data from the in vitro studies were assessed through unpaired Student's t-tests. Each assay was conducted in triplicate and repeated three times. All the cases were two-sided and presented as the mean \pm standard deviation, and $\mathrm{P}<0.05$ was considered to indicate a statistically significant difference.

\section{Results}

FOXM1 expression in OSCC and clinicopathological features. To study the association between FOXM1 expression and clinicopathological characteristics, the expression of FOXM1 in 119 primary OSCC samples was assessed by IHC. All positive cases demonstrated clear nuclear localization of FOXM1 protein (Fig. 1). Among the 119 tumours analysed, 63 (52.94\%) demonstrated high FOXM1 expression and the others exhibited low expression. Collectively, the levels of FOXM1 expression and their association with clinicopathological characteristics are summarized in Table I. Among the 119 patients included in the present study, 28 (23.7\%) experienced local recurrence following surgery, and 20 of the 28 (71.4\%) patients who experienced local recurrence exhibited high FOXM1 expression. Among the 91 patients who did not experience recurrence, $47.25 \%$ (43/91) had high expression levels of FOXM1. Statistically, high expression of FOXM1 was significantly associated with recurrence following surgery $(\mathrm{P}=0.025 ; \mathrm{n}=119)$. No significant association was identified between the FOXM1 protein level and other clinicopathological characteristics, including age at diagnosis, sex, tumour size, clinical TNM classification, pathological grade, cancer location, or lymph and distant metastasis.

FOXM1 expression and survival rates. To determine the association between FOXM1 protein expression and prognostic markers in patients with OSCC, the overall and disease-free survival rates of patients with OSCC were analysed using the log-rank test, and the trends were assessed using the Kaplan-Meier method (Fig. 2). The survival rates of patients with OSCC following radical surgery were computed. During a median follow-up time of 37.3 months (range, 3-81 months), 28 patients $(23.53 \%)$ succumbed to their diseases. Of these 28 patients who succumbed to OSCC, three patients (10.7\%) demonstrated low FOXM1 protein levels, whereas 25 patients (89.3\%) had high FOXM1 protein levels. The survival rate analysis demonstrated that FOXM1 expression was significantly associated with the overall (log-rank, $\mathrm{P}=0.009$ ) and disease-free survival rates of patients with OSCC (log-rank, $\mathrm{P}=0.005$ ).

Next, univariate survival rate analysis was performed to evaluate the independent prognostic factors of tumour recurrence, including TNM classification $(\mathrm{P}=0.033)$, lymphatic metastasis $(\mathrm{P}=0.028)$, distant metastasis $(\mathrm{P}=0.002)$ and FOXM1 expression ( $\mathrm{P}=0.027$; Table II). Multivariate survival rate analysis was performed to evaluate the independent prognostic factors of tumour recurrence, and distant metastasis and FOXM1 were identified to be independent prognosis factors of tumour recurrence in patients with OSCC (Table III). 
Table I. Association between FOXM1 and patient characteristics.

\begin{tabular}{|c|c|c|c|c|}
\hline Characteristic & Low FOXM1 expression $(n=56)$ & High FOXM1 expression $(n=63)$ & $\chi^{2}$ & P-value \\
\hline Age at diagnosis & & & 2.489 & 0.115 \\
\hline$<60$ years & 33 & 28 & & \\
\hline$\geq 60$ years & 23 & 35 & & \\
\hline Sex & & & 0.047 & 0.828 \\
\hline Female & 26 & 28 & & \\
\hline Male & 30 & 35 & & \\
\hline Tumour size & & & 1.739 & 0.419 \\
\hline$<2 \mathrm{~cm}$ & 10 & 7 & & \\
\hline$>2 \mathrm{~cm},<4 \mathrm{~cm}$ & 33 & 36 & & \\
\hline$>4 \mathrm{~cm}$ & 13 & 20 & & \\
\hline TNM classification & & & 0.988 & 0.320 \\
\hline I or II & 30 & 28 & & \\
\hline III or IV & 26 & 35 & & \\
\hline Lymphatic metastasis & & & 1.303 & 0.254 \\
\hline With & 20 & 29 & & \\
\hline Without & 36 & 34 & & \\
\hline Distant metastasis & & & 1.905 & 0.168 \\
\hline With & 3 & 8 & & \\
\hline Without & 53 & 55 & & \\
\hline Local recurrence & & & 5.023 & 0.025 \\
\hline With & 8 & 20 & & \\
\hline Without & 48 & 43 & & \\
\hline Histological grading & & & 0.930 & 0.628 \\
\hline Grade I & 33 & 37 & & \\
\hline Grade II & 18 & 23 & & \\
\hline Grade III & 5 & 3 & & \\
\hline OSCC location & & & 1.895 & 0.595 \\
\hline Tongue & 18 & 27 & & \\
\hline Buccal & 16 & 14 & & \\
\hline Gingiva & 11 & 13 & & \\
\hline $\begin{array}{l}\text { Other (e.g., hard palate } \\
\text { or mouth floor) }\end{array}$ & 11 & 9 & & \\
\hline
\end{tabular}

FOXM1, forkhead box protein M1; TNM, tumour, node and metastasis; OSCC, oral squamous cell carcinoma.
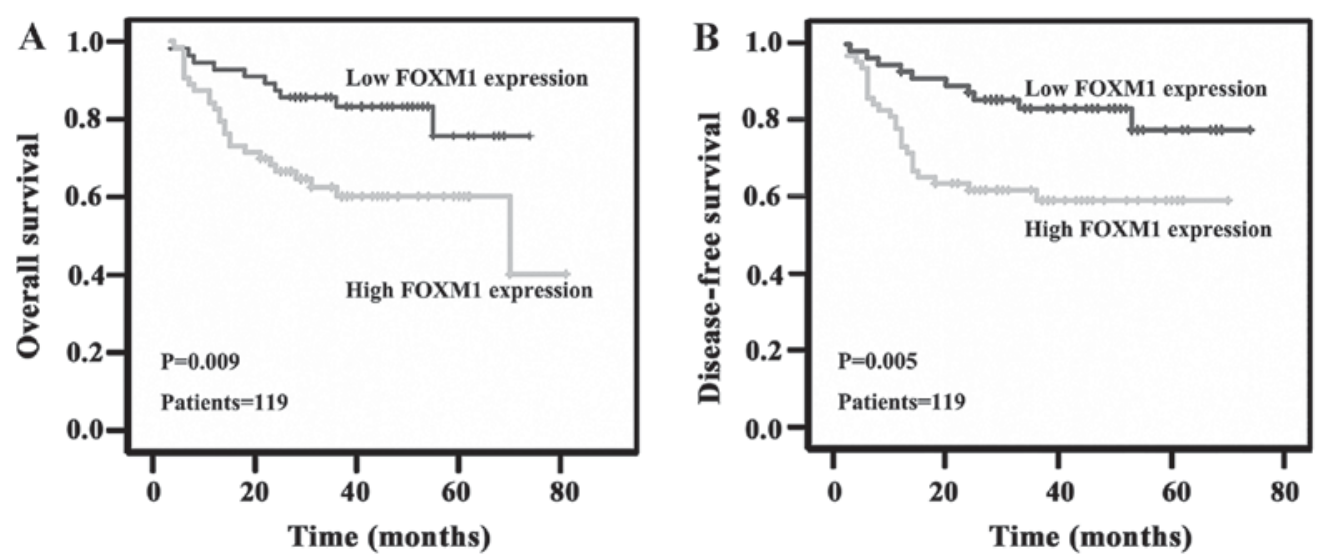

Figure 2. FOXM1 expression significantly correlates with reduced OSCC patient survival. Kaplan-Meier curves of (A) overall and (B) disease-free survival rates of oral squamous cell carcinoma patients with low and high FOXM1 expression. FOXM1, forkhead box protein M1. 
Table II. Summary of survival analysis by univariate Cox regression analysis.

Disease-free survival overall

Parameter

category

Subcategory

P-value

$95 \% \mathrm{CI}$

TNM classification

Lymphatic metastasis

I-II vs. III-IV

0.033

$\operatorname{Exp}(\mathrm{B})$

1.075-5.344

Distant metastasis

with vs. without

0.028

2.397

$1.094-5.013$

with vs. without

0.002

2.342

1.671-10.462

FOXM1 expression

Low vs. high

0.027

4.181

1.121-6.937

CI, confidence interval; TNM, tumour, node and metastasis; FOXM1, forkhead box protein M1.

Table III. Summary of survival analysis by multivariate Cox regression analysis.

Disease-free survival overall

\begin{tabular}{|c|c|c|c|c|}
\hline \multirow{2}{*}{$\begin{array}{l}\text { Parameter } \\
\text { category }\end{array}$} & \multirow[b]{2}{*}{ Subcategory } & \multicolumn{3}{|c|}{$\underline{4}$} \\
\hline & & P-value & $\operatorname{Exp}(B)$ & $95 \% \mathrm{CI}$ \\
\hline TNM classification & I-II vs. III-IV & 0.532 & 1.521 & $0.409-5.657$ \\
\hline Lymphatic metastasis & with vs. without & 0.661 & 1.337 & $0.365-4.898$ \\
\hline Distant metastasis & with vs. without & 0.025 & 3.174 & $1.154-8.735$ \\
\hline FOXM1 expression & Low vs. high & 0.030 & 2.767 & $1.106-6.924$ \\
\hline
\end{tabular}

CI, confidence interval; TNM, tumour, node and metastasis; FOXM1, forkhead box protein M1.

Knockdown of FOXM1 expression by siRNA decreases the proliferation and migration of OSCC cells. To assess whether FOXM1 enhances the malignant phenotypes of OSCC cells, the HN12 and Cal-27 cell lines were selected as the cell models in our preliminary experiments because they highly expressed FOXM1 among certain other cell lines, including HN4, HN6, Tca-811 and SCC-22B (data not shown). FOXM1 siRNA was transiently transfected into two OSCC-derived cell lines, HN12 and Cal-27, the two of which express high FOXM1 protein levels, as demonstrated by western blot analysis (Fig. 3). The transfection of FOXM1 siRNA markedly decreased FOXM1 expression in HN12 and Cal-27 cells (Fig. 3).

The inhibition level of FOXM1 expression was associated with the proliferation of OSCC cells. The results of the present study demonstrated that the silencing of FOXM1 significantly reduced the cellular proliferative capacity compared with the negative control cells (Fig. 4A). The migratory capacity of OSCC cells following siRNA transfection was further examined using in vitro migration and wound-healing assays. As demonstrated in Fig. 4B, following FOXM1 knockdown, significantly lower numbers of HN12 and Cal-27 cells migrated through the Transwell membrane $(\mathrm{P}<0.01$, respectively). The wound healing assay demonstrated that the healing capacity of HN12 and Cal-27 cells was reduced following transfection with FOXM1 siRNA (Fig. 5). These data demonstrated that the inhibition of FOXM1 led to decreased cancer cell proliferation and migration.

FOXM1 inhibition alters the expression of proteins involved in epithelial-mesenchymal transition (EMT) in OSCC cells. To better understand the molecular mechanisms through which FOXM1 contributes to cell migration, the expression levels

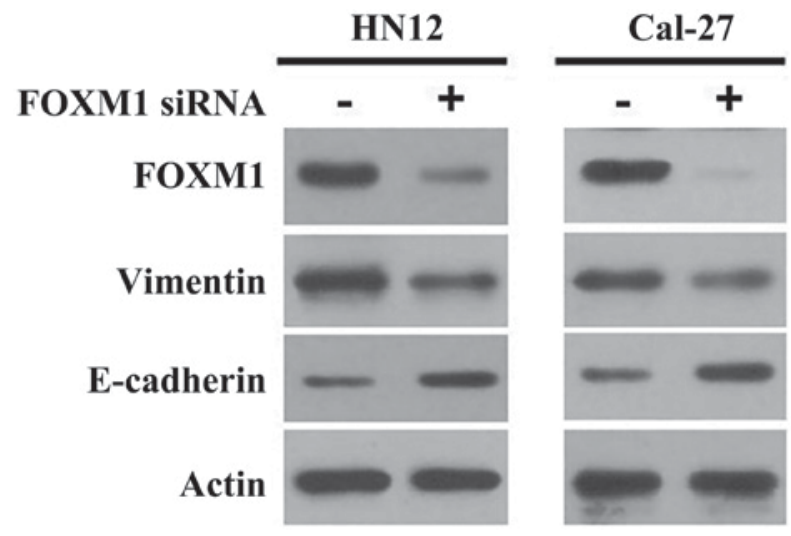

Figure 3. Knockdown of FOXM1 by siRNA alters the expression of proteins involved in epithelial-mesenchymal transition HN12 and Cal-27 cells. FOXM1, forkhead box protein M1; si, small interfering.

of E-cadherin and vimentin protein in FOXM1-knockdown HN12 and Cal-27 cells were determined by western blot analysis. The knockdown of FOXM1 in OSCC cells by siRNA decreased the expression of vimentin and increased the expression of E-cadherin (Fig. 3). As E-cadherin and vimentin are the most important proteins associated with EMT, the results suggested that FOXM1 may regulate the mesenchymal phenotype of OSCC cells.

\section{Discussion}

FOXM1 serves an important regulatory role in a wide range of biological processes, including cell proliferation, cycle 


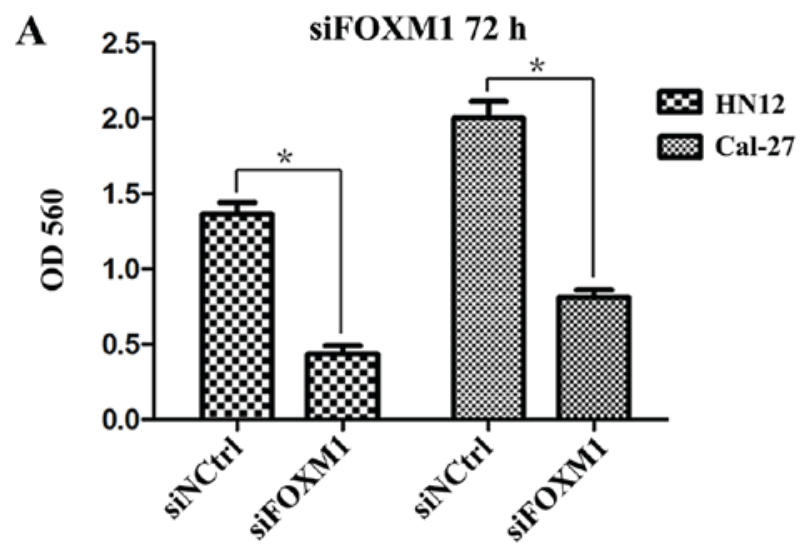

B HN12 siFOXM1 $72 \mathrm{~h}$ migration transwell assay

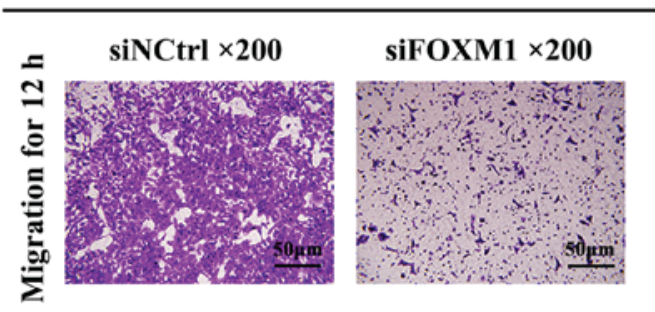

HN12 migration for $12 \mathrm{~h}$

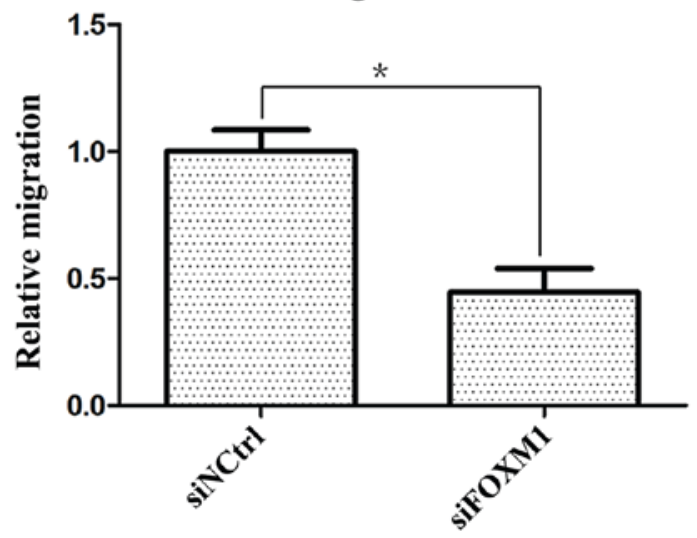

Cal-27 siFOXM1 $72 \mathrm{~h}$ migration transwell assay

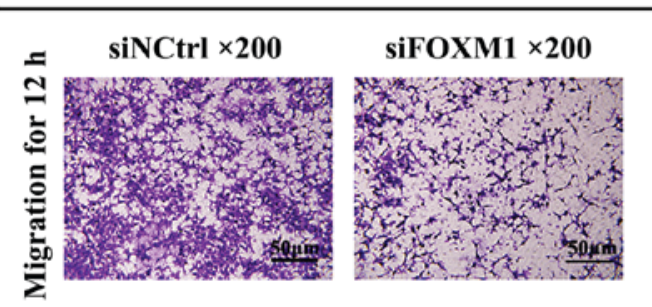

Cal-27 migration for $12 \mathrm{~h}$

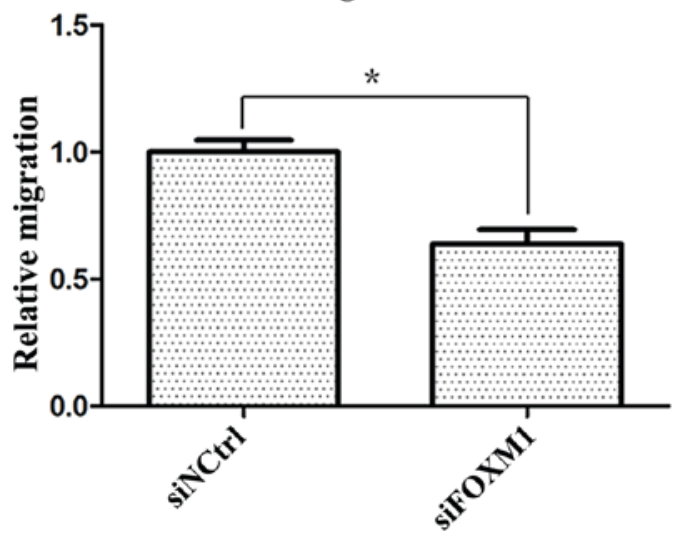

Figure 4. FOXM1 inhibition lowers cell proliferation and migration. (A) A sulforhodamine B assay was performed to evaluate the proliferative capabilities of HN12 and Cal-27 cells transfected with siFOXM1 or siNCtrl. (B) Transwell assays were performed to evaluate the migratory capabilities of HN12 and Cal-27 cells transfected with siFOXM1 or siNCtrl. Top panels: The migratory cells were stained using crystal violet and counted. Bottom panels: The results were quantified by calculating the OD of the crystal violet dye. The data in (A) and (B) are expressed as the mean \pm standard deviation of three independent experiments. " $\mathrm{P}<0.05$. FOXM1, forkhead box protein M1; si, small-interfering; OD, optical density; NCtrl, negative control.

A

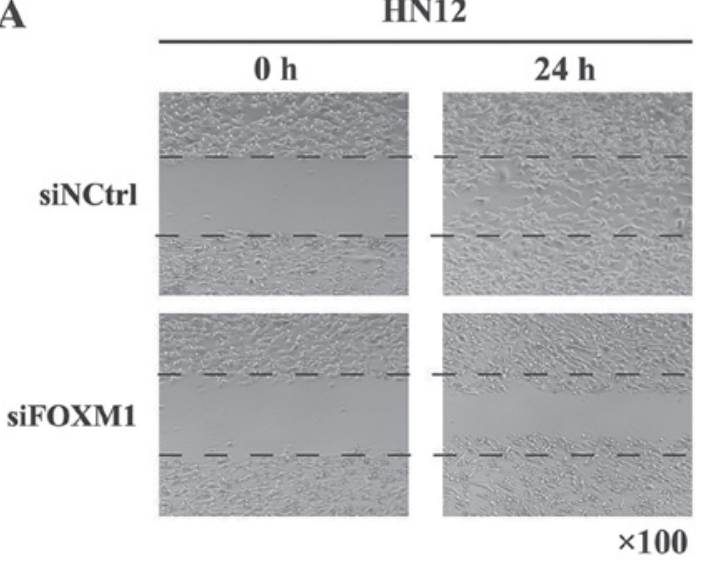

B

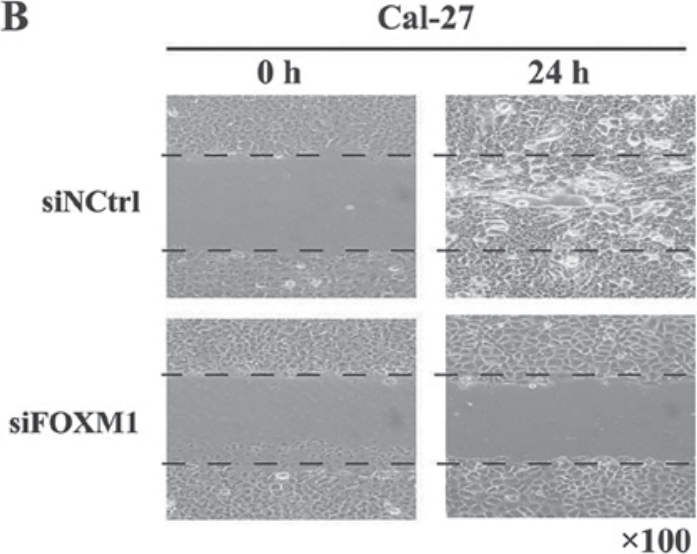

Figure 5. Effect of FOXM1 knock-down on migration. Wound-healing assays were performed to evaluate the migratory capabilities of (A) HN12 and (B) Cal-27 cells transfected with siFOXM1 or a siNCtrl. si, small-interfering; FOXM1, forkhead box protein M1; NCtrl, negative control. 
progression, differentiation, DNA damage repair, tissue homeostasis, angiogenesis and apoptosis (25). The full gene expression profiles of various cancer types have independently and consistently identified FOXM1 as one of the most common highly expressed genes $(13,14)$. A previous study reported that FOXM1 forms part of the set of genes that is upregulated in early tumour development (5). FOXM1 is associated with cancer initiation, progression and metastasis, and drug resistance (4,10-12). FOXM1 is also involved in tumorigenesis by stimulating cell proliferation and cell cycle progression. In oral cancer, FOXM1 and its expression are markedly associated with the tumorigenesis and prognosis of HNSCC (26).

FOXM1 induces tumour occurrence by accelerating human epithelial stem cell regeneration and withstanding differentiation. It has been reported that tobacco is a major risk factor for OSCC development, since the nicotine in tobacco may increase the differentiation of human oral keratinocytes induced by FOXM1 (5). FOXM1B induces correlated genomic variables, including genome-wide loss of heterozygosity (LOH) and copy-number variations, and FOXM1 target genes have been identified within recurrent LOH loci (27). It has been identified that FOXM1 may give methylome a tumour-like structure by prompting methylome recombination in primary human keratinocyte cells (5). However, the clinicopathological importance of FOXM1 in OSCC has not been sufficiently investigated.

In the present study, the expression level of FOXM1 protein was determined in order to better understand the association of FOXM1 expression in patients with OSCC with clinicopathological characteristics and prognosis. The majority $(71.4 \%$; 20/28) of the patients who experienced local tumour recurrence had high expression levels of FOXM1, whereas a lower proportion (43/91) of patients without recurrence demonstrated high FOXM1 expression. This result revealed that FOXM1 is markedly associated with local OSCC recurrence and survival rates in patients with OSCC. In vitro, the knockdown of FOXM1 not only decreased the proliferation, mobility and migration of OSCC cells, but also altered the expression of proteins involved in EMT.

EMT promotes the conversion of epithelial cells to migratory mesenchymal phenotypic cells by breaking junctions among cells, which leads to a low cell-cell adhesion capacity and high cancer aggressiveness $(28,29)$. Tumour cells acquire migratory and invasive capabilities not only by reducing cell adhesions, but also by recombining the cytoskeleton and forming migratory protrusions (30). However, the formation of protrusions, the function of certain organelles and the cellular molecular mechanisms in the EMT process remain to be elucidated. The regulation of the EMT process includes low expression of E-cadherin, which is frequently identified in the majority of epithelial cancers, accompanied by high expression of vimentin, which serves as a marker of the mesenchymal phenotype (29). In the present study, OSCC cells presented a lower E-cadherin level and a higher vimentin level, indicating that patients with OSCC who require more radical therapies may have a poor prognosis. It was previously reported that grainyhead-like 2 regulates FOXM1 expression in OSCC, and thereby regulates homeostasis and cell proliferation (31). Zinc-finger E-box binding homeobox 1 (ZEB1) and other transcriptional regulators, including ZEB2/SIP1, snail family transcriptional repressor (Snail)1, Snail2/Slug, twist family bHLH transcription factor 1 and transcription factor 3, have been identified to be crucial for the EMT process (32). FOXM1 overexpression induces the EMT process and acquisition of the cancer stem cell phenotype, and these effects may be partly regulated by microRNA-200b and easily attenuated by genistein (33). Hypoxia induces EMT in OSCC cells by triggering Notch signalling, and the inhibition of Notch signalling may result in enhanced anti-tumour effects (34). The inhibition of FOXM1 expression is able to directly downregulate the expression of a variety of tumour-promoting factors, including cyclin B1, cell division cycle 25 homologue $\mathrm{B}$ and survivin (35). These factors are usually the key factors in the signalling pathway, and the downregulation of their expression blocks the signalling pathway. The simultaneous inhibition of multiple signalling pathways to antagonize EMT may exert synergistic effects, and may benefit the treatment and prognosis of OSCC. The present study identified that FOXM1 may regulate EMT, but no significant association between FOXM1 expression and metastasis was observed, which may be due to the fact that FOXM1 expression was associated with local invasiveness of the tumours. Possible omissions of occult lymphatic metastasis in the pathology may also be a limitation. In addition, the sample size was small, and a larger sample analysis may be required.

The present study identified that FOXM1 may mediate the OSCC EMT phenotype. The siRNA-mediated knockdown of FOXM1 increased the E-cadherin level and decreased the vimentin level. The effect of FOXM1 on the EMT phenotype may be associated with local OSCC recurrence, and this finding warrants further investigation.

\section{Acknowledgements}

Not applicable.

\section{Funding}

The present study was supported by the National Natural Science Foundation of China (grant no. 81600908), the Jiangsu Provincial Medical Innovation Team (grant no. CXTDA2017036) and the Jiangsu Provincial Medical Youth Talent (grant no. QNRC2016854). The funders had no role in the study design, data collection and analysis, decision to publish or preparation of the manuscript.

\section{Availability of data and materials}

The datasets used and/or analyzed during the current study are available from the corresponding author on reasonable request.

\section{Authors' contributions}

XMZ and HMW conceived and designed the study and proofread the manuscript. YDL and XD wrote the manuscript and performed the experiments, statistical evaluations, and clinical studies. HMD, YNW and HQL performed the clinical studies and statistical evaluations. All the authors read and approved the final manuscript. 


\section{Ethics approval and consent to participate}

The present study was performed with permission from the ethical committee of the Affiliated Stomatological Hospital of Nanjing Medical University. Informed consent was obtained from each patient.

\section{Patient consent for publication}

All patients gave written informed consent before participation in this study.

\section{Competing interests}

The authors declare that they have no competing interests.

\section{References}

1. Torre LA, Bray F, Siegel RL, Ferlay J, Lortet-Tieulent J and Jemal A Global cancer statistics, 2012. CA Cancer J Clin 65: 87-108, 2015.

2. Jerjes W, Upile T, Petrie A, Riskalla A, Hamdoon Z, Vourvachis M, Karavidas K,Jay A,Sandison A,ThomasGJ,etal:Clinicopathological parameters, recurrence, locoregional and distant metastasis in 115 T1-T2 oral squamous cell carcinoma patients. Head Neck Oncol 2: 9, 2010.

3. Carvalho AL, Nishimoto IN, Califano JA and Kowalski LP: Trends in incidence and prognosis for head and neck cancer in the United States: A site-specific analysis of the SEER database. Int J Cancer 114: 806-816, 2005.

4. Ahmad A, Wang Z, Kong D, Ali S, Li Y, Banerjee S, Ali R and Sarkar FH: FoxM1 down-regulation leads to inhibition of proliferation, migration and invasion of breast cancer cells through the modulation of extra-cellular matrix degrading factors. Breast Cancer Res Treat 122: 337-346, 2010.

5. Gemenetzidis E, Bose A, Riaz AM, Chaplin T, Young BD, Ali M, Sugden D, Thurlow JK, Cheong SC, Teo SH, et al: FOXM1 upregulation is an early event in human squamous cell carcinoma and it is enhanced by nicotine during malignant transformation. PLoS One 4: e4849, 2009.

6. Lam EW, Brosens JJ, Gomes AR and Koo CY: Forkhead box proteins: Tuning forks for transcriptional harmony. Nat Rev Cancer 13: 482-495, 2013.

7. Sorlie T, Perou CM, Tibshirani R, Aas T, Geisler S, Johnsen H, Hastie T, Eisen MB, van de Rijn M, Jeffrey SS, et al: Gene expression patterns of breast carcinomas distinguish tumor subclasses with clinical implications. Proc Natl Acad Sci USA 98: 10869-10874, 2001.

8. Tian S, Wang C and An MW: Test on existence of histology subtype-specific prognostic signatures among early stage lung adenocarcinoma and squamous cell carcinoma patients using a Cox-model based filter. Biol Direct 10: 15, 2015.

9. Yu CP, Yu S, Shi L, Wang S, Li ZX, Wang YH, Sun CJ and Liang J: FoxM1 promotes epithelial-mesenchymal transition of hepatocellular carcinoma by targeting Snai1. Mol Med Rep 16: 5181-5188, 2017.

10. Park HJ, Costa RH, Lau LF, Tyner AL and Raychaudhuri P: Anaphase-promoting complex/cyclosome-CDH1-mediated proteolysis of the forkhead box M1 transcription factor is critical for regulated entry into S phase. Mol Cell Biol 28: 5162-5171, 2008.

11. Raychaudhuri P and Park HJ: FoxM1: A master regulator of tumor metastasis. Cancer Res 71: 4329-4333, 2011.

12. Leung TW, Lin SS, Tsang AC, Tong CS, Ching JC, Leung WY, Gimlich R, Wong GG and Yao KM: Over-expression of FoxM1 stimulates cyclin B1 expression. FEBS Lett 507: 59-66, 2001.

13. Myatt SS and Lam EW: The emerging roles of forkhead box (Fox) proteins in cancer. Nat Rev Cancer 7: 847-859, 2007.

14. Koo CY, Muir KW and Lam EW: FOXM1: From cancer initiation to progression and treatment. Biochim Biophys Acta 1819: 28-37, 2012.

15. Ma J, Qi G, Xu J, Ni H, Xu W, Ru G, Zhao Z, Xu W and He X: Overexpression of forkhead box M1 and urokinase-type plasminogen activator in gastric cancer is associated with cancer progression and poor prognosis. Oncol Lett 14: 7288-7296, 2017.
16. Jiang LZ, Wang P, Deng B, Huang C, Tang WX, Lu HY and Chen HY: Overexpression of Forkhead Box M1 transcription factor and nuclear factor- $\mathrm{kB}$ in laryngeal squamous cell carcinoma: A potential indicator for poor prognosis. Hum Pathol 42: 1185-1193, 2011.

17. Abdeljaoued S, Bettaieb I, Nasri M, Adouni O, Goucha A, El Amine O, Boussen H, Rahal K and Gamoudi A: Overexpression of FOXM1 Is a potential prognostic marker in male breast cancer. Oncol Res Treat 40: 167-172, 2017.

18. Wittekind $\mathrm{C}$ and Oberschmid B: TNM classification of malignant tumors 2010: General aspects and amendments in the general section. Pathologe 31: 333-334, 336-338, 2010 (In German).

19. Kim HE, Krug MA, Han I, Ensley J, Yoo GH, Forman JD and Kim HR: Neutron radiation enhances cisplatin cytotoxicity independently of apoptosis in human head and neck carcinoma cells. Clin Cancer Res 6: 4142-4147, 2000.

20. Patel V, Ramesh A, Traicoff JL, Baibakov G, Emmert-Buck MR, Gutkind JS and Knezevic V: Profiling EGFR activity in head and neck squamous cell carcinoma by using a novel layered membrane Western blot technology. Oral Oncol 41: 503-508, 2005.

21. Xiao C, Wang L, Zhu L, Zhang C and Zhou J: Secreted frizzledrelated protein 2 is epigenetically silenced and functions as a tumor suppressor in oral squamous cell carcinoma. Mol Med Rep 10: 2293-2298, 2014

22. Zhu J, Wu YN, Zhang W, Zhang XM, Ding X, Li HQ, Geng M, Xie ZQ and Wu HM: Monocarboxylate transporter 4 facilitates cell proliferation and migration and is associated with poor prognosis in oral squamous cell carcinoma patients. PLoS One 9: e87904, 2014.

23. Zhu Z, Xu X, Yu Y, Graham M, Prince ME, Carey TE and Sun D: Silencing heat shock protein 27 decreases metastatic behavior of human head and neck squamous cell cancer cells in vitro. Mol Pharm 7: 1283-1290, 2010.

24. Wang IC, Chen YJ, Hughes D, Petrovic V, Major ML, Park HJ, Tan Y, Ackerson T and Costa RH: Forkhead box M1 regulates the transcriptional network of genes essential for mitotic progression and genes encoding the SCF (Skp2-Cks1) ubiquitin ligase. Mol Cell Biol 25: 10875-10894, 2005.

25. Nandi D, Cheema PS, Jaiswal N and Nag A: FoxM1: Repurposing an oncogene as a biomarker. Semin Cancer Bilo 52: 74-84, 2018

26. Hwang S, Mahadevan S, Qadir F, Hutchison IL, Costea DE, Neppelberg E, Liavaag PG, Waseem A and Teh MT: Identification of FOXM1-induced epigenetic markers for head and neck squamous cell carcinomas. Cancer 119: 4249-4258, 2013.

27. Teh MT, Gemenetzidis E, Chaplin T, Young BD and Philpott MP: Upregulation of FOXM1 induces genomic instability in human epidermal keratinocytes. Mol Cancer 9: 45, 2010.

28. Tosi GM, Marigliani D, Romeo N and Toti P: Disease pathways in proliferative vitreoretinopathy: An ongoing challenge. J Cell Physiol 229: 1577-1583, 2014.

29. Casaroli-Marano RP,Pagan R and Vilaró S: Epithelial-mesenchymal transition in proliferative vitreoretinopathy: Intermediate filament protein expression in retinal pigment epithelial cells. Invest Ophthalmol Vis Sci 40: 2062-2072, 1999.

30. Yilmaz M and Christofori G: EMT, the cytoskeleton, and cancer cell invasion. Cancer Metastasis Rev 28: 15-33, 2009.

31. Chen W, Yi JK, Shimane T, Mehrazarin S, Lin YL, Shin H, Kim RH, Park NH and Kang MK: Grainyhead-like 2 regulates epithelial plasticity and stemness in oral cancer cells. Carcinogenesis 37: 500-510, 2016.

32. Thierauf J, Veit JA and Hess J: Epithelial-to-mesenchymal transition in the pathogenesis and therapy of head and neck cancer. Cancers (Basel) 9: pii: E76, 2017.

33. Bao B, Wang Z, Ali S, Kong D, Banerjee S, Ahmad A, Li Y, Azmi AS, Miele L and Sarkar FH: Over-expression of FoxM1 leads to epithelial-mesenchymal transition and cancer stem cell phenotype in pancreatic cancer cells. J Cell Biochem 112: 2296-2306, 2011.

34. Ishida T, Hijioka H, Kume K, Miyawaki A and Nakamura N: Notch signaling induces EMT in OSCC cell lines in a hypoxic environment. Oncol Lett 6: 1201-1206, 2013.

35. Nakamura S, Hirano I, Okinaka K, Takemura T, Yokota D, Ono T, Shigeno K, Shibata K, Fujisawa S and Ohnishi K: The FOXM1 transcriptional factor promotes the proliferation of leukemia cells through modulation of cell cycle progression in acute myeloid leukemia. Carcinogenesis 31: 2012-2021, 2010.

This work is licensed under a Creative Commons Attribution-NonCommercial-NoDerivatives 4.0 International (CC BY-NC-ND 4.0) License. 